\title{
An anisotropic micro-sphere-based approach for fiber orientation adaptation in soft tissue
}

\author{
Pablo Sáez, Estefanía Peña, Manuel Doblaré and Miguel Ángel Martínez
}

\begin{abstract}
Evolutionary processes in biological tissue, such as adaptation or remodeling, represent an enterprising area of research. In this work we present a multi-scale model for the remodeling of fibered structures, such as bundles of collagen fibrils. With this aim, we introduce a von Mises statistical distribution function to account for the directional dispersion of the fibrils and we remodel the underlying fibrils by changing their orientation. To numerically compute this process we make use of the micro-sphere approach, which provides a useful multiscale tool for homogenizing the micro-structure behavior, related to the fibrils of the bundle, in the macro-scale of the problem. The results show how the fibrils respond to the stimulus by reorientation of their structure. This process leads to a stiffer material eventually reaching a stationary state. These results are in agreement with those reported in the literature and they characterize the adaptation of biological tissue to external stimuli.
\end{abstract}

Remodeling, micro-sphere, biological tissue, hyperelasticity, anisotropy

\section{INTRODUCTION}

Remodeling and growth are important evolutionary processes in biological tissue on which large amount of research has been focused during recent years. We refer to $(1 ; 2)$ for a review of concepts such as remodeling, growth and morphogenesis. To give a brief overview of these processes we take the definition of growth as the variation of mass via the increase/decrease of the number or size of cells. See, e.g the works of $(3 ; 4 ; 5)$. Morphogenesis defines changes in the shape of the tissue and remodeling defines changes in the micro-structure by reorganization and/or synthesizing of the constituents with negligible changes in the total organ volume. Although these processes can occur simultaneously, we will describe herein a model for the remodeling of fibered soft tissue via reorientation of the fibrils that make up the tissue.

In particular we will study the remodeling of collagen. Collagen, as the main bearing structure in many biological tissues, is very sensitive to remodeling. It is composed of three $\alpha$-chains coiled up in a helical-like structure which reassembles again to create the so called collagen fibril. The reorientation process is known to be driven by both mechanics and biochemical quantities. Strain or stress have been proposed as possible mechanical stimuli. In this work we assume a general mechanical stimulus that will drive the reorientation.

P. Saez, E. Peña, M. Doblare and M. A. Martinez are with the Group of Structural Mechanics and Materials Modeling. Aragón Institute of Engineering Research (I3A), Universidad de Zaragoza, Zaragoza, 50010 Spain and the CIBER-BBN. Centro de Investigación Biomédica en Red en Bioingeniería, Biomateriales y Nanomedicina. e-mail: psaez@unizar.es.

Manuscript received April 19, 2005; revised January 11, 2007.
During the last few years several remodeling models have been proposed. Some of these treat fibers, as 1D elements as in (6), where a consistent linearization was presented in a finite element context. In (7) the eight-chain model (8) was used to model reorientation in living tissue. Moreover, the study presented an experimental test of reorientation of collagen fibers when a mechanical load was applied. Reorientation in biological structures has also been reported in (9) in the context of adaptation of endothelial cells to shear stress, and (10) reported the reorientation of blood vessel fibers due to an increase in blood pressure.

To take into account the micro-structure of the tissue, in (11) the information regarding the dispersion of fibrils was introduced by means of a von Mises statistical distribution function. In (13) a Bingham distribution was presented to characterize the statistical orientation blood vessel fiber, and some comparative studies were carried out. In this contribution, we will use the von Mises distribution for such a purpose. These micro-structural issues have motivated numerous multi-scale approaches (see e.g. (16) among many others). The microsphere-based approach $(17 ; 14 ; 16)$ presents is a novel and promising multi-scale technique for the study of fibered tissue that will be used in this work.

Moreover, the mechanics of fibrils has also gained attention. The Worm-like Chain model (WLC) has been used for modeling the DNA double helix (see e.g (18)) and subsequently adopted for elastomer in (8) and for biological tissue in (7). It was recently used in (16) in the micro-sphere framework to characterize the passive behavior of blood vessels and in (19) for remodeling. Here we will extend the approximation of (16) to include remodeling of fibrils. Other authors have used the spread exponential model proposed in (12). We will adopt herein this latter phenomenological approach. The inclusion of the micro-structure information, both the mechanics of the fibrils and their dispersion, within the micro-sphere approach allows a more detailed analysis of the adaptation process. We will focus on these aspects in this contribution.

\section{MATERial MODEL}

The model presented follows the classical kinematic treatment of finite strains and incompressible materials. Let $\mathbf{X} \in$ $\Omega_{0}$ be a material point in the reference configuration, $\mathbf{x} \in \Omega$ the same point in the spatial configuration $\Omega$ and $\varphi$ the motion mapping. The gradient of $\varphi$ with respect to $\mathbf{X}$ is known as the deformation gradient tensor $\mathbf{F}=\nabla_{\mathbf{X}} \varphi$. The deformation gradient and the Cauchy-Green tensor $\mathbf{C}=\mathbf{F}^{T} \cdot \mathbf{F}$ may be decoupled to account for the volumetric and isochoric parts in 


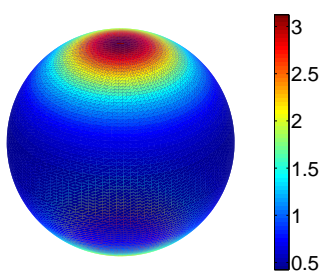

(a) ODF for $b=1$

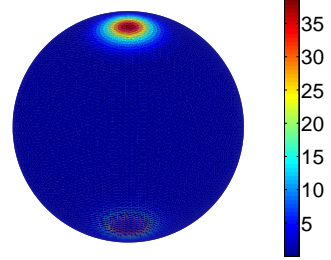

(b) ODF for $b=10$.
Fig. 1. Shape of the von Mises ODF for $b=1$ and $b=10$.

the standard manner

$$
\begin{aligned}
& \mathbf{F}=\left[J^{1 / 3} \mathbf{I}\right] \cdot \overline{\mathbf{F}}, \\
& \mathbf{C}=\left[J^{2 / 3} \mathbf{I}\right] \cdot \overline{\mathbf{C}},
\end{aligned}
$$

with $J^{1 / 3} \mathbf{I}$ and $J^{2 / 3} \mathbf{I}$ tensors associated with changes in volume and $\overline{\mathbf{F}}$ and $\overline{\mathbf{C}}$ with the isochoric deformation. I represents the second order unit tensor. Let $\mathbf{r}$ now be a vector in the reference configuration. Its associated isochoric counterpart in the current configuration $\overline{\mathbf{t}} \in \Omega$ is given by the push-forward operation as $\overline{\mathbf{t}}=\overline{\mathbf{F}} \cdot \mathbf{r}$.

As usual in Continuum Mechanics of anisotropic fibered biological tissue, we will make use of a Strain Energy Density Function (SEDF) in decoupled form: volumetric and isochoric parts, while the latter is decoupled again into isotropic and anisotropic parts respectively

$$
\Psi=\Psi_{\mathrm{vol}}+\Psi_{\mathrm{iso}}+\Psi_{\mathrm{ani}}
$$

with $\Psi_{\mathrm{vol}}(\mathrm{J})$ the SEDF associated to the volumetric part, $\Psi_{\text {iso }}\left(\mathrm{I}_{1}\right)$ to the isotropic contribution, associated to the extracellular matrix modeled here by a Neo-Hookean constitutive model as $\Psi_{\text {iso }}=\mu\left[I_{1}-3\right] . J=\operatorname{det}(\mathbf{C}), \mu$ a material parameter and $\mathrm{I}_{1}=\operatorname{tr}(\mathbf{F}) . \Psi_{\text {ani }}$ is related to the anisotropic contribution of the tissue, associated in our case to the collagen fibers.

To account for the anisotropy of the tissue we use the von Mises distribution $(\rho)$ which establishes the dispersion of the fibrils around a preferential direction of anisotropy, a. Note that this function present mid-plane symmetry and rotational symmetry properties around the preferential direction $\mathbf{a}$. The $\pi$-periodic von Mises function (4) has been adopted in this work.

$$
\rho(\theta)=4 \sqrt{\frac{b}{2 \pi}} \frac{\exp (b[\cos (2 \theta)+1])}{\operatorname{erfi}(\sqrt{2 b})},
$$

where $b \in \mathbb{R}^{+}$denotes the so called concentration parameter. When $b \rightarrow 0$, Eq. 4 leads to an isotropic material, while $b \rightarrow \infty$ represents a transversely isotropic state. $\operatorname{Erfi}(x)$ is the imaginary error function approximated by a sufficiently large number of terms by means of its MacLaurin series expansion.

$$
\operatorname{erfi}(x) \approx \pi^{-1 / 2}\left[2 x+\frac{2 x^{3}}{3}+\sum_{j=3}^{k} \frac{x^{2 j-1}}{a(j)}\right],
$$

with $a(j)=0.5[2 j-1][j-1]$ !. Eq. 5 provides sufficient accuracy for values of $b \leq 20$ with expansions of at least 60 terms. Fig. 1 shows the representation of two distributions for different values of $b$.
Moving to the fibril behavior, we set an exponential-type SEDF, that may be written together with its derivative as

$$
\psi\left(\bar{\lambda}_{i}\right)=\left\{\begin{array}{ccc}
0 & \text { if } & \bar{\lambda}_{i}<1 \\
\frac{k_{1}}{2 k_{2}}\left[\exp \left(k_{2}\left[\bar{\lambda}_{i}^{2}-1\right]^{2}-1\right)\right] & \text { if } & \bar{\lambda}_{i} \geq 1
\end{array}\right.
$$

$$
\partial_{\bar{\lambda}_{i}} \psi\left(\bar{\lambda}_{i}\right)=\left\{\begin{array}{ccc}
0 & \text { if } & \bar{\lambda}_{i}<1 \\
2 k_{1} \bar{\lambda}_{i}\left[\bar{\lambda}_{i}^{2}-1\right] \exp \left(k_{2}\left[\bar{\lambda}_{i}^{2}-1\right]^{2}\right) & \text { if } & \bar{\lambda}_{i} \geq 1
\end{array}\right.
$$

with $k_{1}$ and $k_{2}$ material parameters and $\bar{\lambda}_{i}=\left\|\overline{\mathbf{t}}_{i}\right\|$. As this is derived from Eq. 6, the fibrils do not bear any load under compression. We obtain the Piola-Kirchhoff and Kirchhoff stress tensors from Eq. 6 as

$$
\begin{aligned}
& \mathbf{S}_{i}=2 \partial_{\mathbf{C}} \psi\left(\bar{\lambda}_{i}\right)=2 \bar{\lambda}_{i}^{-1} \partial_{\bar{\lambda}_{i}} \psi\left(\bar{\lambda}_{i}\right) \mathbf{r}_{i} \otimes \mathbf{r}_{i} \\
& \boldsymbol{\tau}_{i}=\mathbf{F} \cdot \mathbf{S}_{i} \cdot \mathbf{F}^{t}=2 \bar{\lambda}_{i}^{-1} \partial_{\bar{\lambda}_{i}} \psi\left(\bar{\lambda}_{i}\right) \overline{\mathbf{t}}_{i} \otimes \overline{\mathbf{t}}_{i}
\end{aligned}
$$

The micro-sphere approach homogenizes by no more than averaging over the unit sphere the variable of interest $(\bullet)$. To numerically integrate such an average, this integral is approximated through a discretization of $(m)$ discrete orientation vectors and the corresponding weight factors $w^{i}(17)$ as

$$
\langle(\bullet)\rangle=\frac{1}{4 \pi} \int_{\mathbb{U}^{2}}(\bullet) \mathrm{dA} \approx \sum_{i=1}^{m} w^{i}(\bullet)_{i}
$$

Despite the numerical aspect of the micro-sphere approach, the integration directions can be interpreted as the orientations of the fibrils within the collagen bundle. In Fig. 2 we present the fibril distributions shown in Fig. 1, with a more physical interpretation.

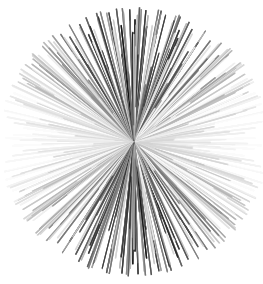

(a) Fibrils for $b=1$.

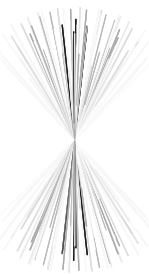

(b) Fibrils for $b=10$.
Fig. 2. Fibril representation of the statistical distributions of Fig 1.

The averaged SEDF and Kirchhoff stress are then expressed as:

$$
\begin{gathered}
\Psi_{\mathrm{ani}}=\frac{1}{4 \pi} \int_{\mathbb{U}^{2}} n \rho \psi(\bar{\lambda}) d A \approx \sum_{i=1}^{m} n \rho_{i} w^{i} \psi\left(\bar{\lambda}_{i}\right) \\
\boldsymbol{\tau}_{\mathrm{ani}}=\frac{1}{4 \pi} \int_{\mathbb{U}^{2}} n \rho \boldsymbol{\tau} d A \approx \sum_{i=1}^{m} n \rho_{i} \psi^{\prime}\left(\bar{\lambda}_{i}\right) \bar{\lambda}_{i}^{-1} \overline{\mathbf{t}}_{i} \otimes \overline{\mathbf{t}}_{i} w^{i}
\end{gathered}
$$

with $\Psi_{\text {ani }}$ and $\psi$ the SEDF in the macro and fiber levels respectively, $\rho_{i}$ the probabilistic value for each integration direction and $n$ the fibrils number density. 


\section{REORIENTATION PROCESS}

The reorientation process is provided by appropriate rate equations in the spirit of $(6 ; 19)$. These reorientation equations that define the evolution of the integration directions, will also undergo a change in the distribution shape. Following the approaches adopted by the latter authors, for 1D fibers and the micro-sphere approach respectively, the spatial velocity gradient and its symmetric and antisymmetric part are given by

$$
\mathbf{l}=\dot{\mathbf{F}} \cdot \mathbf{F}^{-1}=\mathbf{d}+\boldsymbol{\omega}
$$

The time derivative of $\mathbf{r}_{i}$ is a rotation with angular velocity vector $\boldsymbol{\omega}_{i}$,

$$
\begin{aligned}
& \dot{\mathbf{r}}_{\mathbf{i}}=\boldsymbol{\omega}_{i} \times \mathbf{r}_{i} \text {, with } \\
& \boldsymbol{\omega}_{i}:=\mathbf{r}_{i} \times \boldsymbol{\Xi}_{3}
\end{aligned}
$$

we obtain the rate of $\mathbf{r}_{i}$ as

$$
\dot{\mathbf{r}_{i}}=\left[\mathbf{I}-\mathbf{r}_{i} \otimes \mathbf{r}_{i}\right] \cdot \boldsymbol{\Xi}_{3}
$$

where $\boldsymbol{\Xi}$ describes a matrix of eigenvectors of a given general quantity. Therefore $\boldsymbol{\Xi}_{I}$, with $I=1,2,3$, represents the minimum, medium and maximum eigenvectors respectively. We make the supposition that the fibrils reorient towards the maximum principal direction $\boldsymbol{\Xi}_{3}$, e.g. a strain measure. In order to update precisely the vector $\mathbf{r}_{i}$, we use the exponential map (see $(20 ; 6)$ and references therein), adopting an explicit update, leading to

$$
\mathbf{r}_{i}^{n+1}=\exp \left(\hat{\boldsymbol{\omega}}_{i}^{n} \Delta t\right) \cdot \mathbf{r}_{i}^{n}
$$

where $\hat{\boldsymbol{\omega}}_{i}{ }^{n}=-\boldsymbol{\varepsilon} \cdot \boldsymbol{\omega}_{i}{ }^{n}$ is the associated skew symmetric tensor and $\varepsilon$ the third-order permutation tensor.

\section{RESULTS}

In this Section we will discuss an example of the model described above. We set $k_{1}=20, k_{2}=5, b=1$, similar to the values reported in (16). We take the time step $\Delta t=0.1$ and apply a uniaxial test to our model, with a deformation gradient $\mathbf{F}=1 / \sqrt{\lambda} \mathbf{e}_{x} \otimes \mathbf{e}_{x}+1 / \sqrt{\lambda} \mathbf{e}_{y} \otimes \mathbf{e}_{y}+\lambda \mathbf{e}_{z} \otimes \mathbf{e}_{z}$, up to $\lambda=1.5$. Moreover we will suppose that the preferential direction is pre-oriented along the direction of stretching, that is $\mathbf{e}_{z}$. To visualize the evolution of the position of the fibrils, we present the fibril distribution in Fig. 3 for different times, leading to a very concentrated distribution of the fibrils at the end of the process.

Fig. 4(a) presents the evolution of the stress in the stretching direction, showing the asymptotic behavior expected, where the reorientation reaches a stationary state. However, biological structures have many different mechanical properties that change their passive and active behavior. To extend the results, we also provide results for a softer material, with $k_{2}=4$ and the others parameters as described above. When the ratio between the matrix stiffness and fiber stiffness decreases the remodeling occurs in a more gradual way as shown in Fig. 4(b).In fact, the process presents the most realignment for the initial isotropic material $(b=0)$, as presented in (19), and almost no realignment for very concentrated fibrils $(b \rightarrow \infty)$.

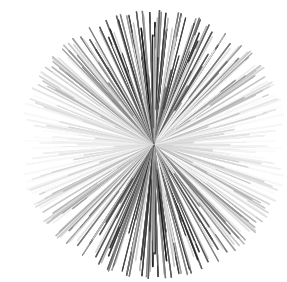

(a) Fibrils distribution at $\mathrm{t}=0$

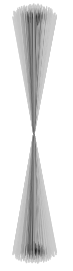

(c) Fibrils distribution at $\mathrm{t}=25$

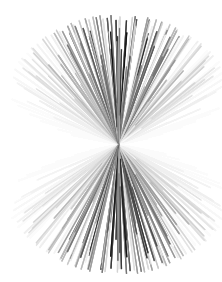

(b) Fibrils distribution at $\mathrm{t}=5$

$$
\mid
$$

(d) Fibrils distribution at $\mathrm{t}=100$
Fig. 3. Distribution of the fibrils at different times of the analysis, starting from a concentration parameter $b=1$. The figures show how the concentration evolves during the reorientation process, leading at $t=100$ to an almost $1 \mathrm{D}$ fiber.

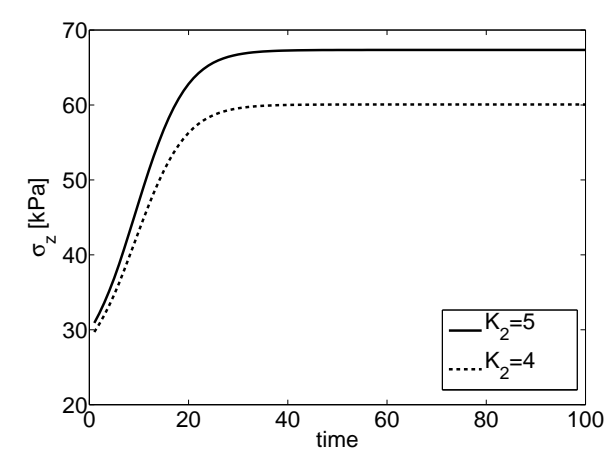

Fig. 4. Evolution of stresses in $\mathbf{e}_{z}$ as the adaptation evolves .

\section{DISCUSSION}

This work presents a $3 \mathrm{D}$ reorientation model for fibered biological tissue to take into account the change of shape of this kind of structures. Most previous works treat this issue in a unidimensional way and only a few of them account for information of the micro-structure $(21 ; 22)$. By means of the anisotropic micro-sphere-based approach, we have been able to carry out a multi-scale model to take into account the micro-structural behavior in the macro-scale. Moreover, we have been able to give a more physical interpretation of the integration directions, associating them to the fibrils of the collagen bundle, and subsequently constructing a 3D structure of the bundle. Given this micro-structure, we were able to compute the reorientation of the fibrils.

Despite the possibilities presented by our model for remodeling and other evolving processes such as growth, some limitations should be pointed out. The first, is the experimental determination of those parameters related with the fibril reorientation. This will be our primary goal in the future. Besides, the mean direction should be allowed to rotate to align with the maximum principal direction, which will give much more flexibility to the model presented. Another important 
aspect to take into account is the use of different driving mechanical stimuli. Many other variations and new material parameters could be included to fit more realistic situations, but the goal herein is to present a methodology for anisotropic materials and not to provide a complete sensitivity analysis of the behavior of the model.

\section{CONCLUSIONS}

We have proposed a novel 3D reorientation model of fibered structures. The model captures the adaptation of biological tissue by means of orientation of the fibrils due to a mechanical stimulus via reorientation. We have been able to capture the stiffening evolution of the tissue in the direction of the orientation.

\section{ACKNOWLEDGEMENTS}

Support of the Spanish Ministry of Research and Innovation through the research project DPI2010-20746-C03-01 and through the grant BES-2009-028593, as well as the support of the Instituto de Salud Carlos III through the CIBER initiative are highly appreciated.

\section{REFERENCES}

[1] L. A. Taber, "Biomechanics of growth, remodeling, and morphogenesis," Appl. Mech. Rev., vol. 48, pp. 487-545, 1995.

[2] J. D. Humphrey, "Need for a continuum biochemomechanical theory of soft tissue and cellular growth and remodeling," in Biomechanical Modelling at the Molecular, Cellular and Tissue Levels. Springer Vienna, 2009.

[3] E. K. Rodriguez, A. Hoger, and A. D. McCulloch, "Stress-dependent finite growth in soft elastic tissues," $J$ Biomech, vol. 27, no. 4, pp. 455-467, Apr. 1994.

[4] J. D. Humphrey and K. R. Rajagopal, "A constrained mixture model for growth and remodeling of soft tissues," Math Models Methods Appl Sci, vol. 12, no. 3, pp. 407-430, 2002.

[5] E. Kuhl, A. Menzel, and P. Steinmann, "Computational modeling of growth - a critical review, a classification of concepts and two new consistent approaches," Comput Mech, vol. 32, no. 1-2, pp. 71-88, 2003.

[6] G. Himpel, A. Menzel, E. Kuhl, and P. Steinmann, "Time-dependent fibre reorientation of transversely isotropic continua - finite element formulation and consistent linearization," Intl J Numer Meth Eng, vol. 73, no. 10, pp. 1413-1433, 2008.

[7] E. Kuhl, K. Garikipati, E. M. Arruda, and K. Grosh, "Remodeling of biological tissue: Mechanically induced reorientation of a transversely isotropic chain network," J Mech Phys Solids, vol. 53, no. 7, pp. 1552-1573, 2005.

[8] E. M. Arruda and M. C. Boyce, "A three-dimensional constitutive model for the large stretch behavior of rubber elastic materials," J Mech Phys Solids, vol. 41, no. 2, pp. 389-412, 1993.

[9] G. Dai, MR. Kaazempur-Mofrad, H. Narayanan, YC. Zhang, B. Blackman, S. Vaughn, BR. Kamm,
G. Garcia-Cardena and MA. Gimbrone "Distinct endothelial phenotypes evoked by arterial waveforms derived from atherosclerosis-susceptible and resistant regions of human vasculature," PNAS, vol. 101, no. 41, pp. 301-312, Sept. 1995.

[10] HM. Finlay, L. Mccullough and PB. Canham "3dimensional Collagen Organization of Human Brain Arteries At Different Transmural Pressures," J. Vasc. Res., vol. 32, no. 5, pp. 14871-14876, Oct. 2004.

[11] T. C. Gasser, R. W. Ogden, and G. A. Holzapfel, "Hyperelastic modelling of arterial layers with distributed collagen fibre orientations," J Roy Soc Interface, vol. 3, pp. 15-35, 2006.

[12] Holzapfel, G. A., Gasser, T. C., Ogden, R. W., 2000. A new constitutive framework for arterial wall mechanics and a comparative study of material models. J Elasticity V61 (1), 1-48.

[13] V. Alastrué, P. Sáez, E. Peña, M. A. Martinez, and M. Doblare, "On the use of the bingham statistical distribution in microsphere-based constitutive models for arterial tissue," Mech Res Commun., vol. 37, no. 8, pp. 700-706, 2010.

[14] C. Miehe, S. Göktepe, and F. Lulei, "A micro-macro approach to rubber-like materials-part i: the non-affine micro-sphere model of rubber elasticity," J Mech Phys Solids, vol. 52, no. 11, pp. 2617-2660, 2004.

[15] F. C. Caner and I. Carol, "Microplane constitutive model and computational framework for blood vessel tissue," $J$ Biomech Eng, vol. 128, no. 3, pp. 419-427, 2006.

[16] V. Alastrué, M. A. Martinez, M. Doblare, and A. Menzel, "Anisotropic micro-sphere-based finite elasticity applied to blood vessel modelling," J Mech Phys Solids, vol. 57, no. 1, pp. 178-203, 2009.

[17] Z. P. Bazant and B. H. Oh, "Microplane model for progressive fracture of concrete and rock," J Eng MechASCE Journal of Engineering Mechanics-asce, vol. 111, no. 4, pp. 559-582, 1985.

[18] C. Bustamante, Z. Bryant, and S. B. Smith, "Ten years of tension: single-molecule DNA mechanics," Nature, vol. 421, no. 6921, pp. 423-427, 2003.

[19] A. Menzel and T. Waffenschmidt, "A microsphere-based remodelling formulation for anisotropic biological tissues," Philos Trans R Soc London Ser A, vol. 367, no. 1902, pp. 3499-3523, 2009.

[20] J. E. Marsden and T. S. Ratiu, Introduction to Mechanics and Symmetry: A Basic Exposition of Classical Mechanical Systems. Springer Verlag, 1999.

[21] F. Baaijens, C. Bouten, and N. Driessen, "Modeling collagen remodeling," J Biomech, vol. 43, no. 1, pp. 166175, 2010.

[22] A. Menzel, M. Harrysson, and M. Ristinmaa, "Towards an orientation-distribution-based multi-scale approach for remodelling biological tissues," Comput Meth Biomech Biomed Eng, vol. 11, no. 5, pp. 505-524, 2008. 